\title{
Particle-core model for transverse dynamics of beam halo
}

\author{
T. P. Wangler, K. R. Crandall,* R. Ryne, and T. S. Wang \\ Los Alamos National Laboratory, P.O. Box 1663, Los Alamos, New Mexico 87545
}

(Received 25 June 1998; published 18 December 1998)

\begin{abstract}
The transverse motion of beam halo particles is described by a particle-core model which uses the space-charge field of a continuous cylindrical oscillating beam core in a uniform linear focusing channel to provide the force that drives particles to large amplitudes. The model predicts a maximum amplitude for the resonantly-driven particles as a function of the initial mismatch. We have calculated these amplitude limits and have estimated the growth times for extended-halo formation as a function of both the space-charge tune-depression ratio and a mismatch parameter. We also present formulas for the scaling of the maximum amplitudes as a function of the beam parameters. The model results are compared with multiparticle simulations and we find very good agreement for a variety of initial particle distributions. [S1098-4402(98)00022-6]
\end{abstract}

PACS numbers: 29.17.+w, 29.27.Bd, 41.75. $-\mathrm{i}$

\section{INTRODUCTION}

High-intensity charged-particle beams can develop extended low-density halos; examples of such halos have been observed at the output of the LANSCE proton linac [1] and have also been studied experimentally [2]. The existence of halos can have serious consequences in particle accelerators. If halo particles are lost in the accelerator, they may induce radioactivity. For the next generation of high intensity proton linac projects, such as accelerator production of tritium [3], it is necessary to obtain a more quantitative understanding of the physics of the halo.

Multiparticle simulation studies of round beams in uniform linear focusing channels have provided some useful physical insights into the dynamics of high-current beams. For a nonequilibrium beam injected with the correctly matched rms size, the initial distribution relaxes with accompanying emittance growth over a time of only about one quarter plasma period to a quasiequilibrium state with an approximately uniform central core in real space and with an edge that falls off over a distance approximately equal to the Debye length [4]. The relative sizes of the central core and the Debye edge depend on whether the beam is emittance or space-charge dominated. In the extreme space-charge limit the Debye edge approaches zero and the spatial density is uniform, whereas in the emittance-dominated limit the profile is dominated by the Debye edge and a spatial density that is approximately Gaussian [5]. In numerical simulations of rms-mismatched beams for both uniform and periodic channels, a tail develops that is much more extended and more densely populated [6-10] than the Debye tail of a matched beam. The simulation results have suggested a simple particle-core model to describe a mismatched

\footnotetext{
*Present address: TechSource, Inc., 301 N. Guadalupe, Santa Fe, NM 87501.
}

beam. In the model the central charge distribution, or core, oscillates as a consequence of the initial mismatch, and particles, including those in the initial Debye edge, are represented by single test particles that interact with the external focusing force and with the time-dependent space-charge field of the core [11,12]. Various aspects of the particle-core model have been studied at several laboratories [13-24]. Perhaps the most significant result obtained from an analytic solution of the model equations [12] has been the discovery that the interaction between the particles and the core results in a parametric resonance that is effective in driving some particles to large amplitudes, and this process is sufficient to account for the halo that is observed in the simulations.

In a typical proton linac after the beam is bunched, the bunches formed have an approximately spheroidal shape with a longitudinal to transverse aspect ratio that ranges from near 1 (spherical) to 3 or 4 . To model the transverse dynamics for beams where the bunch length is several times larger than the radius, we use the simple particlecore model based on transverse particle motion in the space-charge field of a continuous cylindrical beam core whose radius also oscillates because of a mismatch. We have also studied a particle-core model for a spherical bunch [20,21], and the results are qualitatively similar to those that will be presented here; these results will be the subject of a later paper. In the model the spacecharge field of the core is approximated by replacing the unknown beam distribution with that of an equivalentuniform beam, where the equivalent beam has the same rms size as the actual beam. We find that the model results are not sensitive to the detailed choice of the coredensity distribution.

Several aspects of the particle-core model will be emphasized in this paper that have not been given as much attention in previous work. First, we are interested in obtaining numerical results that are useful for linac design work and for comparison with particle simulations. 
Therefore, we solve the nonlinear equations of the model by numerical integration. Second, we note that for practical equilibrium distributions in accelerators with linear focusing forces, excluding the singular KapchinskyVladimirsky (K-V) distribution and excluding the extreme space-charge limit, a tail exists at the beam edge that will usually extend into the region of influence of the resonance. For these beams, K-V core instabilities are not required to explain the existence of particles near the core that can be resonantly excited, although if such instabilities are present $[18,19,21]$, they could feed additional particles into the tail. Thus, we use the model to represent a beam whose initial density distribution includes particles that populate a finite size Debye tail, and the fraction of those particles that are included within the region of influence of the resonance will end up in the halo. Third, we emphasize the model prediction of a maximum amplitude for the resonantly-driven particles as a function of the mismatch, and we identify this maximum amplitude as the maximum size of the halo. Finally, we find that the maximum amplitude is proportional to the matched rms beam size, and using the analytic solution relating the rms size of the beam core to the current, emittance, and focusing strength, we obtain a scaling formula for the size of the transverse beam halo.

\section{PARTICLE-CORE MODEL}

Consider a round continuous beam propagating in a uniform beam transport system with azimuthal symmetry and a linear radial focusing force; such a transport system can be used to represent the average or smoothed behavior of a beam in a quadrupole focusing channel. The central beam core will be assumed to have a uniform spatial density and will be described by the envelope equation. An arbitrary beam particle will experience an external focusing force and a space-charge force, which we will calculate by assuming that the particle interacts only with the core. We approximate the transverse spacecharge force by assuming that the core is an infinitely long uniform cylinder of charge, whose radius oscillates because of an initial mismatch. The space-charge force is linear when the particle is inside the core and nonlinear when outside. We ignore any influence of an individual particle on the motion of the core.

The above assumptions lead to the following model. The transverse equation of motion of the core-radius $R$ is given as a function of axial coordinate $z$ by

$$
\frac{d^{2} R}{d z^{2}}+k_{0}^{2} R-\frac{\varepsilon^{2}}{R^{3}}-\frac{K}{R}=0,
$$

where the focusing force is represented by $k_{0}$, which is also the wave number or phase advance per unit length of the transverse particle oscillations at zero current, $K=q I / 2 \pi \varepsilon_{0} m c^{3} \beta^{3} \gamma^{3}$, where $q, m$, and $\beta c$ are the charge, mass, and axial velocity of the particles, re- spectively, $\gamma$ is the relativistic Lorentz factor, $I$ is the beam current (charge per unit length times axial speed),

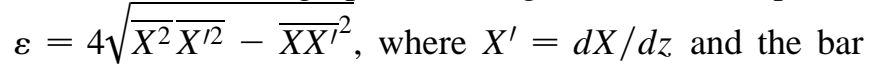
indicates an average over the distribution, is the total unnormalized emittance, $c$ is the speed of light, and $\varepsilon_{0}$ is the permeability of free space. For a matched beam in a uniform focusing channel, $d^{2} R / d z^{2}=0$, and the matched-beam radius is given by $R^{2}=R_{0}^{2}=$ $\left(\varepsilon / k_{0}\right)\left[u+\sqrt{1+u^{2}}\right]$, where $u=K / 2 \varepsilon k_{0}$ is a dimensionless space-charge parameter. If the initial radius is unequal to the matched value $R_{0}, d^{2} R / d z^{2} \neq 0$ and for a round beam a symmetric breathing mode is excited, resulting in oscillations in the radius. The transverse equation of motion of a single particle moving radially in the field of the uniform core is

$$
\frac{d^{2} X}{d z^{2}}+k_{0}^{2} X-F_{\mathrm{sc}}=0,
$$

where $X$ is the radial displacement and $F_{\text {sc }}$ is the spacecharge term given for a uniform density by

$$
F_{\text {sc }}=\left\{\begin{array}{ll}
K X / R^{2}, & |X|<R \\
K / X, & |X| \geq R
\end{array} .\right.
$$

The net focusing force including the space-charge term for a particle that always remains within the core is represented by the wave number $k$, given by $k^{2}=k_{0}^{2}-$ $k_{p}^{2} / 2$, where $k_{p}^{2}=2 K / R_{0}^{2}$ is the square of the plasma wave number. The core breathing-mode wave number $k_{c}$ can be expressed as $k_{c}^{2}=2 k_{0}^{2}+2 k^{2}=4 k^{2}+k_{p}^{2}$.

For the matched case, the core radius is constant, and there is no net change in particle energy averaged over a complete period of the particle motion. For the mismatched case, the core radius oscillates, and particles can either gain or lose energy with each transit through the core. The particles experience a nonlinear force proportional to $K / X$ when they are spatially outside the core and from Gauss's law this force is independent of the instantaneous size of the core. When the particles pass through the core, they are decelerated by the spacecharge force as they approach the core, and accelerated by the space-charge force as they leave the core. The net space-charge impulse delivered to the beam may be described as the sum of a core-entrance contribution plus a core-exit contribution. These impulses may be either diminished or enhanced relative to the matched case, depending on whether the core radius is larger or smaller than the equilibrium value at the time the particle passed through. For example, if a particle enters the core when its radius is larger than the matched value and exits when the radius is smaller than the matched value, a net energy impulse is delivered to the particle. Gluckstern [12] has shown that this motion can be described by a nonlinear parametric resonance; the particles resonate with the core motion when the particle wave number $\nu$ is related to the core breathing-mode wave number $k_{c}$ by $k_{c}=2 \nu$. 
Note that $\nu=k$ for particles that always remain within the core, and $\nu>k$ for particles with amplitude larger than the core radius. For nonzero beam current, one can show that the resonance condition is satisfied only when $\nu>k$, i.e., for particles with amplitudes larger than the core radius. The decrease of the space-charge field with displacement experienced by particles that are outside the core produces an increase of the wave number $\nu$ with amplitude so that the $k_{c}=2 \nu$ resonant condition cannot be maintained as the amplitude increases; this effect limits the resonant amplitude growth.

The two model equations, Eqs. (1) and (2), can be converted to dimensionless form by introducing the following variables: $r=R / R_{0}, x=X / R_{0}$, and $\tau=k_{0} z$. We express the in-core space-charge tune-depression ratio for the particles as $\eta=k / k_{0}=\sqrt{1+u^{2}}-u$. The dimensionless equation of motion of the core becomes

$$
\frac{d^{2} r}{d \tau^{2}}+r-\frac{\eta^{2}}{r^{3}}-\frac{1-\eta^{2}}{r}=0
$$

and the dimensionless single-particle equation is

$$
\frac{d^{2} x}{d \tau^{2}}+x-\left(1-\eta^{2}\right) \times \begin{cases}x / r^{2}, & |x|<r \\ 1 / x, & |x| \geq r\end{cases}
$$

Equations (4) and (5) depend only on the single parameter $\eta$. The matched core corresponds to $r=1$. The divergence $x^{\prime}$ in dimensionless coordinates becomes $x^{\prime}=d x / d \tau=X^{\prime} / k_{0} R_{0}$, and the emittance $\varepsilon$ in dimensionless coordinates becomes $\eta=\varepsilon / k_{0} R_{0}^{2}$. To describe the mismatch of the core radius we need a second parameter, which we define as a mismatch parameter equal to the initial core radius $\mu=r_{\text {initial, }}$, where the initial phasespace ellipse is assumed to be upright.

\section{GRAPHICAL PRESENTATION OF SOLUTIONS}

The differential equations, (4) and (5), for the evolution of the core radius and the trajectory of a single particle can be numerically integrated as functions of $\eta$ and $\mu$. We have integrated these equations numerically for different initial conditions and use three methods to display the results of particle motion: 1) plot the particle trajectory $x$ versus time $t, 2$ ) display the continuous particle trajectory in phase space from $t=0$ to some time $t$, and 3) show a stroboscopic phase-space map of a particle trajectory or an array of particle trajectories. The stroboscopic method accumulates many snapshots of phase space taken periodically at some specified phase of the core oscillation. The stroboscopic map is useful as a method for displaying regions in phase space where classes of trajectories can be identified. Using the stroboscopic map, resonance regions surround stable fixed points, and regions of chaos appear as a random scatter of points.

First, we show in Figs. 1-3 the three plots for the case with $\eta=0.5$ and an initial dimensionless core radius $r=\mu=0.62$, which corresponds to a maximum radius

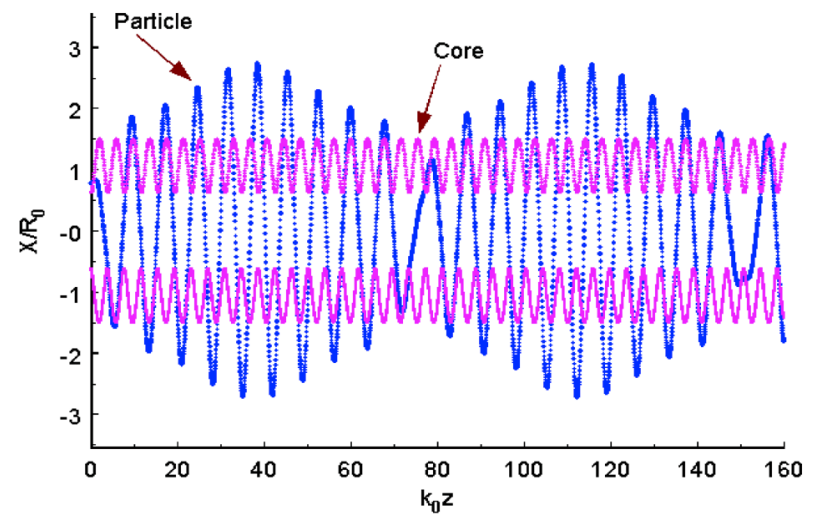

FIG. 1. (Color) Displacement versus time for a particle trajectory with initial coordinates $x=0.77, x^{\prime}=0$, and with $\mu=0.62$ and $\eta=0.5$.

$r=1.5$. We show $x$ versus $t$ in Fig. 1, the continuous phase-space plot in Fig. 2, and the stroboscopic plot taken when the core radius is at its minimum value in Fig. 3, all for a particle with initial coordinates $x=0.77$ and $x^{\prime}=$ 0 , which is within the region of the parametric resonance. The amplitude of this orbit varies from a minimum of 0.77 to a maximum of 2.73. Our choice of the strobe phase, when the core has its minimum radius, corresponds to the time at which the resonant orbits reach their maximum displacements. The maximum of the orbits within the core occurs when the core is at its maximum radius.

Next, we show stroboscopic maps for an initial array of 32 particle coordinates uniformly distributed along the positive horizontal and vertical axes, again at a coreoscillation phase that corresponds to the minimum core radius. Figures 4-8 show the stroboscopic plots for a mismatch parameter $\mu=0.62$ and for $\eta=0.1-0.9$ in steps of 0.2 , all taken at the time when the core radius is minimum. Recall that $\eta=0.1$ is a space-chargedominated beam and $\eta=0.9$ is an emittance-dominated beam. A separatrix with inner and outer branches defines three different regions. First are the quasielliptical curves

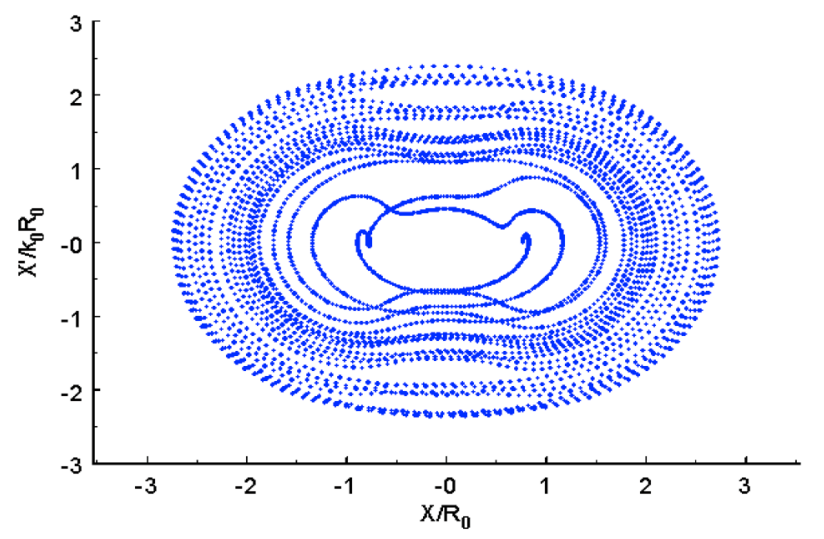

FIG. 2. (Color) Continuous plot of $x$ versus $t$ in phase space for a particle with initial coordinates $x=0.77, x^{\prime}=0$, and with $\mu=0.62$ and $\eta=0.5$. 


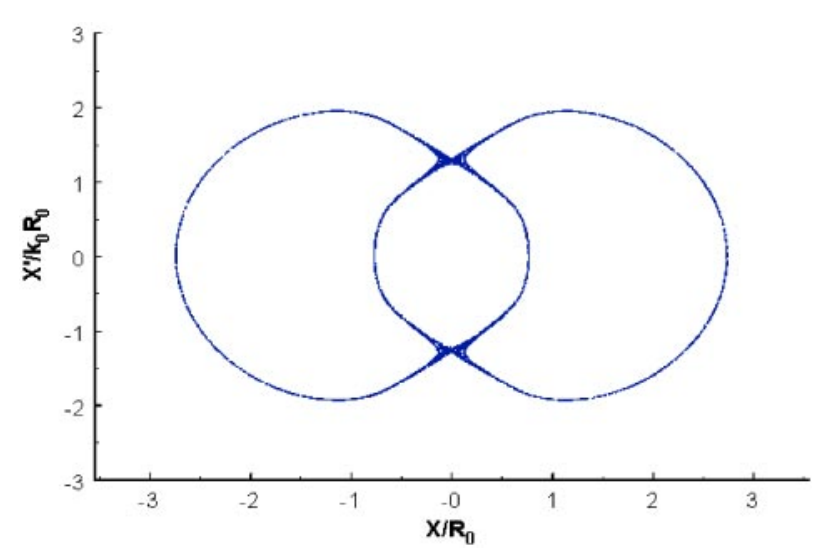

FIG. 3. (Color) Stroboscopic plot for a particle trajectory with initial coordinates $x=0.77, x^{\prime}=0$, and with $\mu=0.62$ and $\eta=0.5$. The points are plotted once per core oscillation at the time when the core radius is minimum. These points correspond to more core cycles than those in Fig. 2.

near the center, including the beam core radius at $r=$ 0.62 , and a small region between the core boundary and the inner separatrix. Second are the two regions of concentric curves centered on the stable fixed points symmetrically located on the $x$ axis. These regions display the parametric resonance trajectories. Finally, there are families of quasielliptical trajectories outside the outer separatrix. For small $\eta \leq 0.3$, breakdown of the separatrix is clearly observed with associated stochastic behavior; the stochastic regime grows as $\eta$ decreases. In this parameter region the motion of the trajectories near the separatrix becomes very sensitive to the initial conditions. For small $\eta$ the stochastic behavior replaces the regular trajectories between the core boundary and the inner separatrix. Stroboscopic plots have been made over the range between $\mu=1.1$ and 3.0. The qualitative appearances of these plots are similar to those at $\mu=0.62$ (or 1.5). The areas occupied by the parametric resonance increase with increasing $\mu$, although

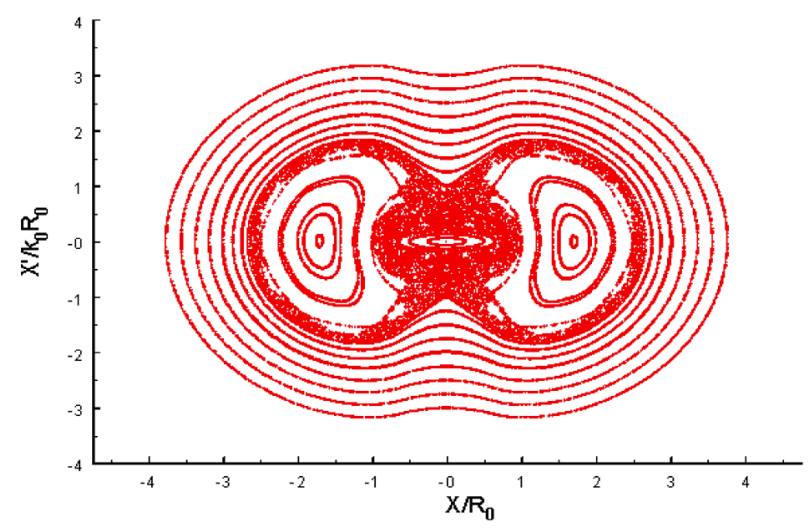

FIG. 4. (Color) Stroboscopic plot for mismatch parameter $\mu=$ 0.62 and tune-depression ratio $\eta=0.1$ for the uniformdensity core.

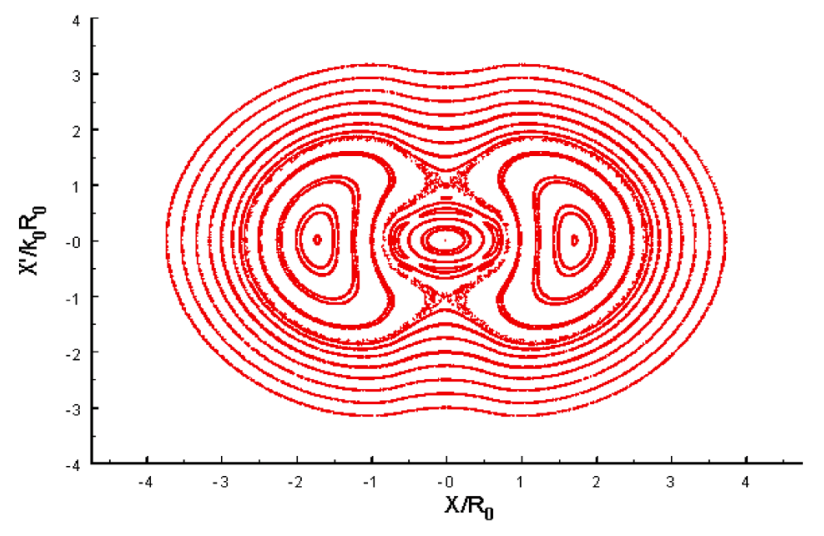

FIG. 5. (Color) Stroboscopic plot for mismatch parameter $\mu=$ 0.62 and tune-depression ratio $\eta=0.3$ for the uniformdensity core.

the positions of the stable fixed points are insensitive to $\mu$; thus, the amplitude variation of the resonant orbits increases as the mismatch parameter $\mu$ deviates from unity.

Figure 9 shows the stroboscopic plot for $\mu=0.62$ and $\eta=0.5$, obtained by replacing the uniform-density core with that of a Gaussian core. Outside the core region, this plot looks very similar to that for the uniform core shown in Fig. 6. The similarity of Figs. 6 and 9 suggests that for resonant partices, the single-particle dynamics are insensitive to the details of the core-density distribution used in the model.

Next, we present our interpretation of the model and its relation to beam halo. We assume that most of the particles that form the halo in the real beam are those that initially populate the Debye edge of the beam that lies outside the inner separatrix and inside the resonance region of the stroboscopic plot. Because the density of the real beam decreases with increasing amplitude, most of these particles will initially lie near the inner separatrix. After some time these particles will be driven outwards

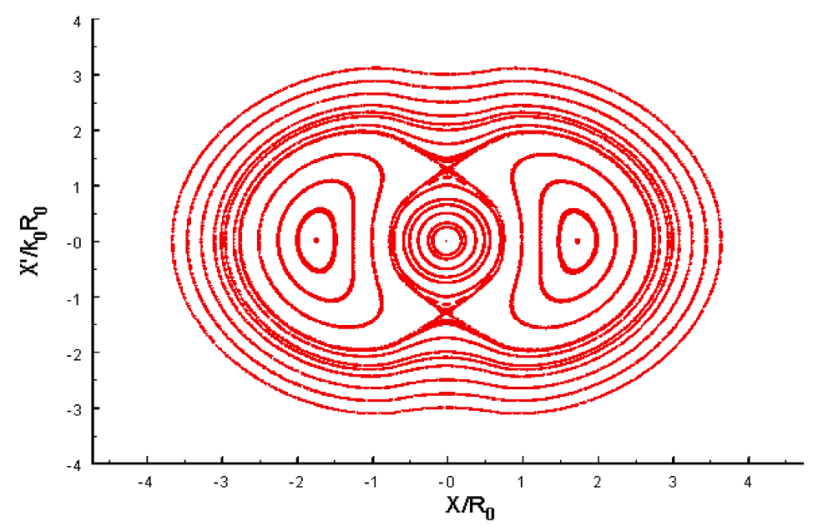

FIG. 6. (Color) Stroboscopic plot for mismatch parameter $\mu=$ 0.62 and tune-depression ratio $\eta=0.5$ for the uniformdensity core. 


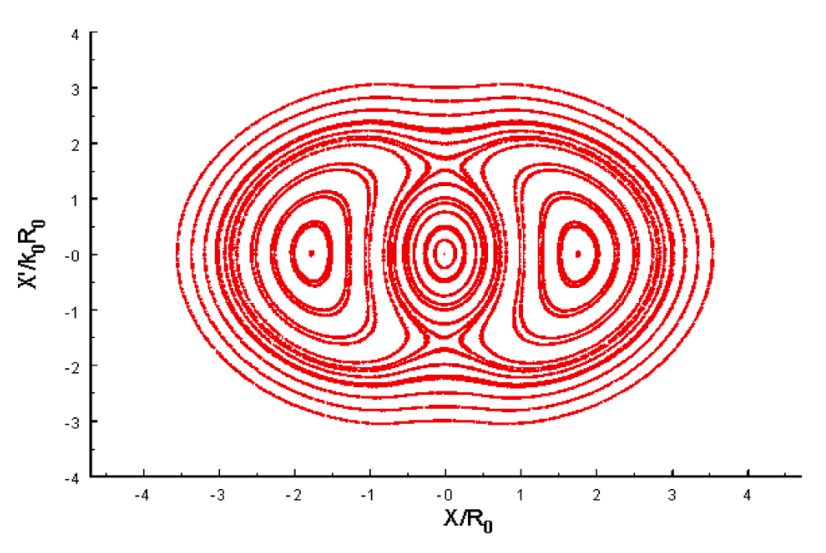

FIG. 7. (Color) Stroboscopic plot for mismatch parameter $\mu=$ 0.62 and tune-depression ratio $\eta=0.7$ for the uniformdensity core.

to the outer separatrix and will thereafter cycle back and forth bounded by the two separatrices. Eventually, after enough phase mixing, these particles form the halo that will be distributed throughout the resonance region, and the outer separatrix will define a maximum amplitude for the halo. This picture is an approximation for at least two reasons. First, if the core distribution is not stable, some particles that are initially within the core may increase the number of particles in the halo. This would be an effect that is beyond the scope of the model, which assumes a fixed core distribution. We find that an unstable core does occur for a K-V beam, as shown in Sect. V, and has been studied by Gluckstern et al. [18,19,21]. Even for these particles we find from simulation that the maximum amplitude is still limited by the outer separatrix. Second, the edge of a real beam may also extend outward across the unstable fixed points on the velocity axis of the stroboscopic plots and produce an initial population of the focusing-dominated region. These particles would have a larger maximum amplitude than that defined by the outer separatrix. Because the particle distribution is

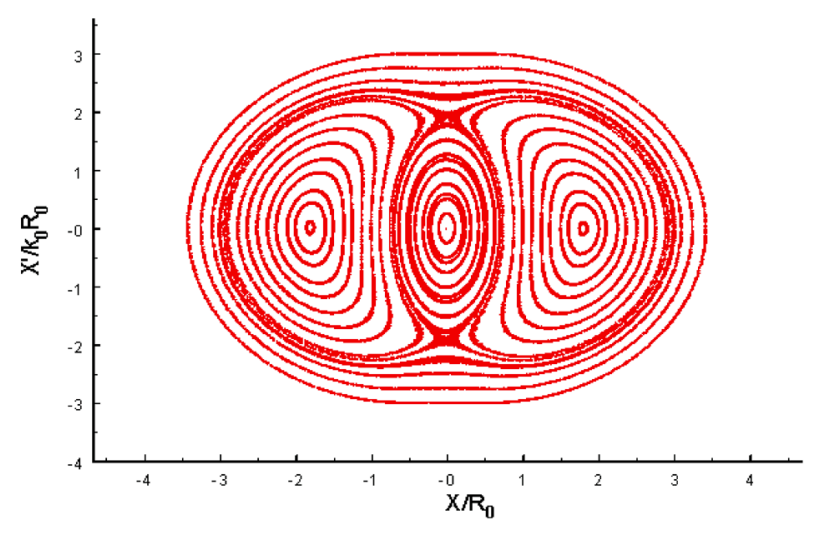

FIG. 8. (Color) Stroboscopic plot for mismatch parameter $\mu=$ 0.62 and tune-depression ratio $\eta=0.9$ for the uniformdensity core. decreasing strongly with increasing transverse velocity, we expect that the corresponding amplitude increase will be small. Consequently, we will assume that the main source of the beam halo is associated with those particles that are initially within the spatial Debye edge of the beam and that lie initially within the resonance region. These particles will be limited by the outer separatrix, which we will associate with the maximum amplitude of the halo. Later, we will test the maximum amplitude predictions by comparison with numerical simulation.

\section{CHARACTERISTICS OF RESONANT TRAJECTORIES}

In the previous section we have seen that orbits in the region of the parametric resonance can undergo large changes in amplitude, and we have argued that these particles constitute the majority of the halo. Figure 10 for $\mu=1.1$ and Fig. 11 for $\mu=1.5$ show the values of four important parameters that characterize the stroboscopic plots for the uniform-density core as a function of the tune-depression ratio $\eta$. Shown are the minimum core radius $r_{\min }$, the coordinate of the stable fixed point $x_{s}$, and the minimum and maximum values, $x_{\min }$ and $x_{\max }$, of the intercepts of the separatrix with the $x$ axis. From Figs. 10 and 11 we see that the values of these dimensionless parameters are very insensitive to $\eta$. As the mismatch parameter $\mu$ deviates further from unity, the difference between $x_{\min }$ and $r_{\min }$ decreases, suggesting that the number of particles in the halo should increase, and the difference between $x_{\max }$ and $x_{\min }$ increases, suggesting that the amplification of the halo amplitude increases.

We can use the model to estimate approximate growth times of the resonant amplitudes that determine the halo. Assuming that the resonance region of stroboscopic phase space nearest the core is initially populated with particles, we are interested in the time it takes for particle amplitudes to grow to a value near $x_{\max }$. However,

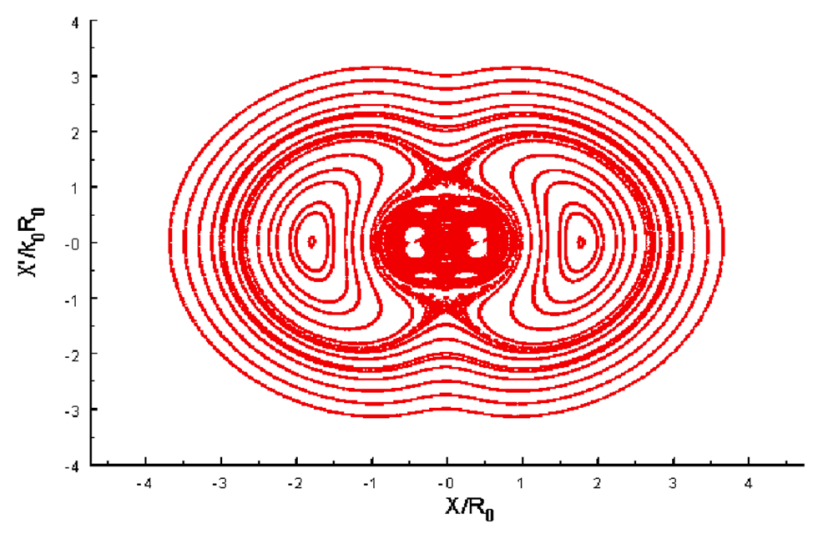

FIG. 9. (Color) Stroboscopic plot obtained by replacing the uniform-density core with a Gaussian-density core of the same rms size. The plot is shown for mismatch parameter $\mu=0.62$ and tune-depression ratio $\eta=0.5$. 


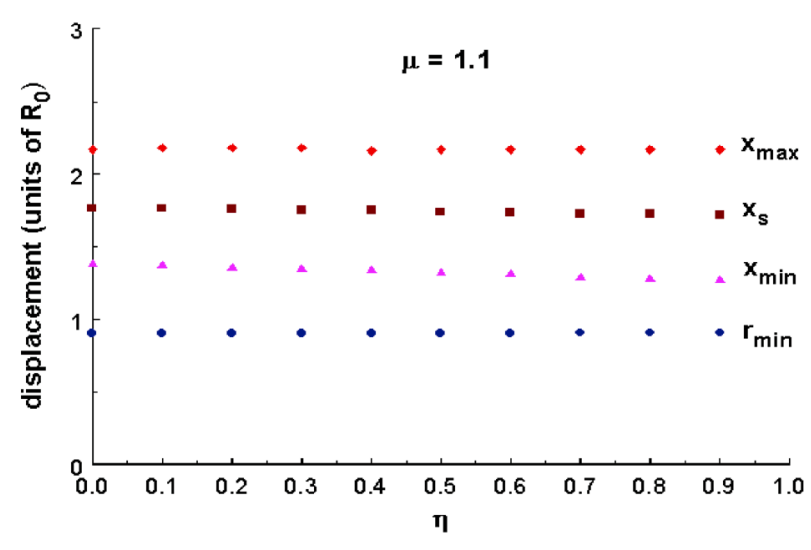

FIG. 10. (Color) Four parameters that characterize the stroboscopic plots as a function of the tune-depression ratio $\eta$ for $\mu=1.1$. Shown are the minimum core radius $r_{\text {min }}$, the coordinate of the stable fixed point $x_{s}$, and the minimum and maximum values of the intercepts of the separatrix with the $x$ axis, $x_{\min }$ and $x_{\max }$, respectively.

because the net restoring force is nonlinear, this time will depend on the amplitude and will be expected to increase as the initial coordinate approaches the inner separatrix. To estimate the time scale for the growth, we have numerically calculated the period for amplitude growth from the minimum to the maximum value, for small initial deviations from the stable fixed point, where the period is nearly independent of the initial deviation. These small amplitude results are plotted in units of core-oscillation cycles in Fig. 12 for mismatch parameters $\mu=1.1,1.5$, and 2.0. Generally, the model predicts that these growth times are insensitive to the mismatch parameter $\mu$ but increase strongly with tune depression $\eta$ for large $\eta$. Comparisons of these growth times from the model to numerical simulation results show that the results in Fig. 12 are of the correct order of magnitude

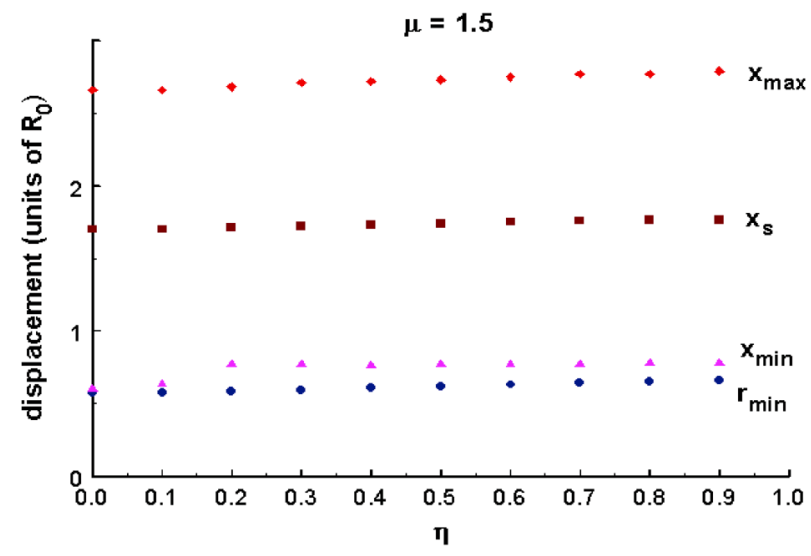

FIG. 11. (Color) Four parameters that characterize the stroboscopic plots as a function of the tune-depression ratio $\eta$ for $\mu=1.5$. Shown are the minimum core radius $r_{\text {min }}$, the coordinate of the stable fixed point $x_{s}$, and the minimum and maximum values of the intercepts of the separatrix with the $x$ axis, $x_{\min }$ and $x_{\max }$, respectively. but, for most cases, agree to within only about a factor of 2. The halo growth times from the simulations depend on the initial particle distribution and are often difficult to define in an unambiguous way.

\section{COMPARISON OF THE MODEL WITH MULTIPARTICLE SIMULATIONS}

We have compared the maximum amplitude predictions of the model with numerical simulation using $10^{4}$ particles per run. Two space-charge codes were used; one uses Gauss's law to calculate the space-charge fields in a cylindrical geometry, and the other, called SCHEFF, which is widely used for linac simulation studies, is based on a particle-in-cell method [25]. Several initial distributions were used, including Gaussian in both position and velocity space (truncated at $3 \sigma$ ), semi-Gaussian (uniform in space and Gaussian in velocity space), and 4D Waterbag (uniformly filled ellipsoid in 4D phase space). For each case we have carried out a set of runs varying the initial mismatch parameter $\mu$. After the beam sizes were set for a given mismatch parameter, the velocities were scaled to produce the same emittance as for the matched case.

In Figs. 13 and 14 the ratio of the maximum particle amplitude from simulation to the rms size of the matched beam is plotted versus the mismatch parameter $\mu$; the lower and upper curves in Fig. 13 are for tune-depression ratios of $\eta=0.5$ and 0.9 . The simulations were run for at least 100 plasma periods, sufficient for the amplitudes to reach an apparent asymptotic value. A typical highpower proton linac may contain a few hundred plasma periods.

The maximum amplitudes from the different spacecharge codes and for all the distributions agree very well with each other and with the cylinder particle-core model. The insensitivity of the maximum amplitude to the initial particle distribution observed in the simulations is

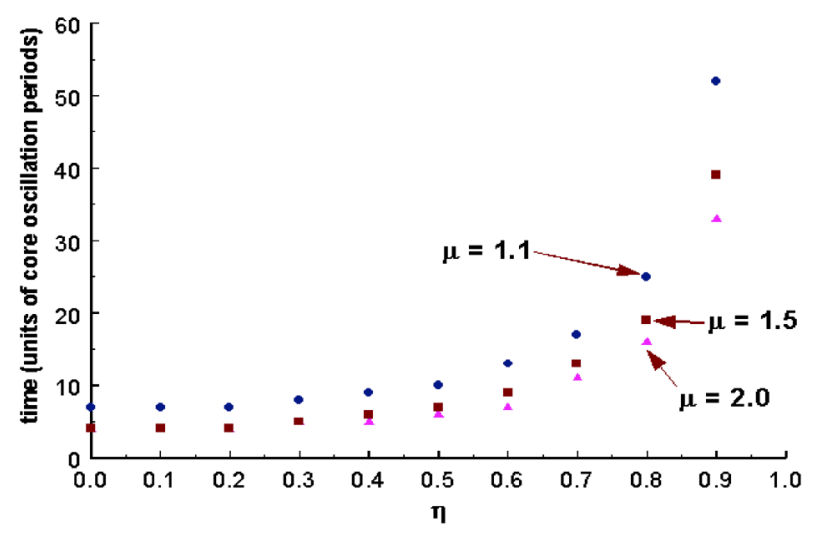

FIG. 12. (Color) Time in core-oscillation cycles for amplitude growth from the minimum to the maximum value for small initial deviations from the stable fixed point, plotted versus the tune depression $\eta$ for mismatch parameters $\mu=1.1,1.5$, and 2.0. 


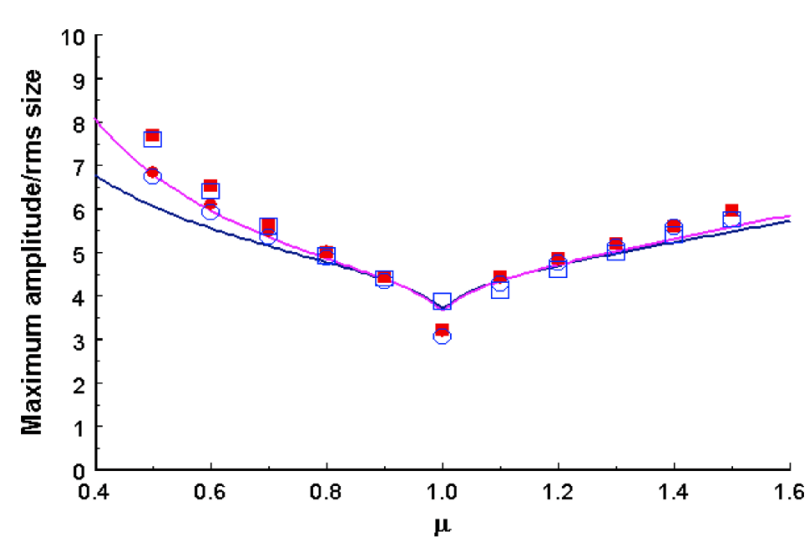

FIG. 13. (Color) Multiparticle simulations comparing the particle-core model with the Gauss-law space-charge code for a round beam in a uniform-focusing channel. The ratio of the maximum amplitude to the matched rms size is plotted versus the mismatch parameter $\mu$. The lower and upper curves are from the particle-core model for tune-depression ratios of $\eta=0.5$ and 0.9 , respectively. The solid and open symbols are for the initial Gaussian and semi-Gaussian distributions, respectively, and the circles and squares are for tune-depression ratios of $\eta=0.5$ and 0.9 .

consistent with the results shown earlier that the particlecore model predictions are insensitive to the choice of uniform- or Gaussian-core distributions.

Figure 15 shows particle simulation results from the Gauss-law space-charge routine for a mismatched $\mathrm{K}-\mathrm{V}$ beam having the same parameters as Fig. 6. Although the initial distribution has the property that it is uniform in phase space, it is unstable for the parameters chosen, and the resulting phase space of Fig. 15 is highly nonuniform. The curve bounding the distribution in Fig. 15 lies just outside the outer separatrix shown in Fig. 6. Thus, even for an unstable core, the maximum particle ampli-

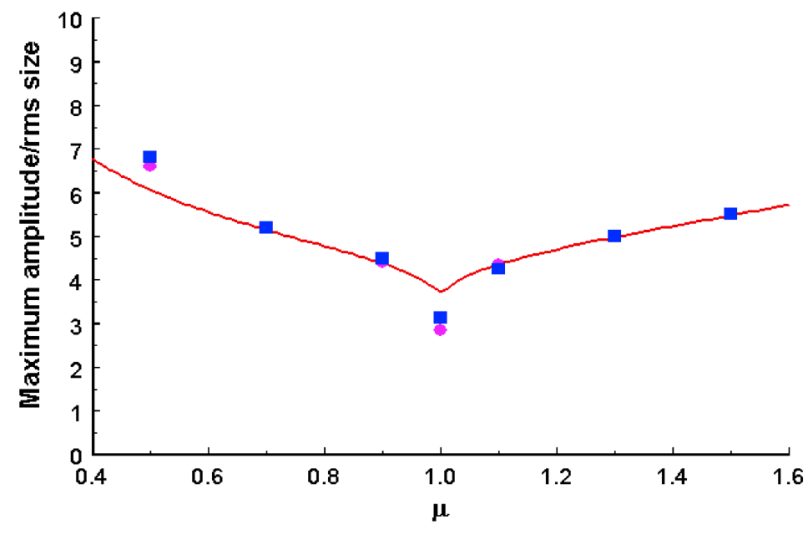

FIG. 14. (Color) Multiparticle simulations comparing the particle-core model (curve) with the Gauss (circles) and SCHEFF (squares) space-charge codes for a tune-depression ratio of $\eta=0.5$. The ratio of the maximum amplitude to the matched rms size is plotted versus the mismatch parameter $\mu$. The initial distribution is a $4 \mathrm{D}$ Waterbag. tude from simulation is in excellent agreement with the maximum amplitude from the particle-core model.

\section{SCALING OF BEAM HALO AMPLITUDES}

We find that the normalized maximum particle amplitude curves from the model, shown in Figs. 13 and 14, are well described over the useful range of tune-depression ratios by an approximate empirical formula

$$
x_{\max } / a=A+B|\ln (\mu)|,
$$

where $x_{\max }$ is the maximum resonant-particle amplitude, $a=R_{0} / 2$ is the matched core rms size, and $A$ and $B$ are weak functions of the tune-depression ratio, approximately given by $A=B=4$ [20]. Equation (6) is not a good approximation for values of $\mu$ very near 1 , where at $\mu=1, x_{\max } / a=2$. Based on Eq. (6), we obtain simple scaling formulas for the maximum particle amplitudes of the mismatched beam. The space-charge parameter introduced in Sect. II can be written as

$$
u=\frac{q \bar{I}}{16 \pi \varepsilon_{0} m c^{2} \gamma^{2} \beta k_{0} \ell f \varepsilon_{n, \mathrm{rms}}} .
$$

The parameters appearing in Eq. (7) include the average beam current $\bar{I}$ for a string of bunches with bunch frequency $f$, the total bunch length $\ell$, and normalized rms input emittance $\varepsilon_{n, \mathrm{rms}}=\beta \gamma \varepsilon / 4$. The average current is related to the number of particles per bunch $N$ and the bunch frequency $f$ by $\bar{I}=q N f$. The particle-core model for an rms-mismatched beam predicts that the halo particles produced by the resonance have a maximum amplitude for a given mismatch. From Eq. (6), the numerical solution predicts that the maximum amplitude is proportional to the matched rms size $a$ of the core given by

$$
a^{2}=\frac{\varepsilon_{n, \mathrm{rms}}}{k_{0} \beta \gamma}\left[\sqrt{1+u^{2}}+u\right]
$$

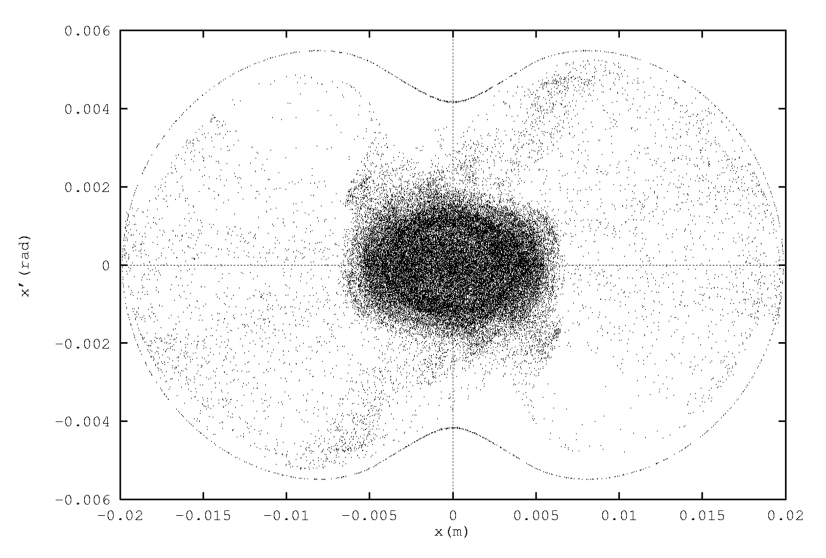

FIG. 15. Beam phase space from a $2 \times 10^{6}$ particle simulation of an initial K-V distribution. The outer peanut-shaped curve lies just outside the outer separatrix of the particle-core model shown in Fig. 6. 
In the space-charge dominated limit, when $u \gg 1$, the rms beam size is

$$
a^{2} \cong \frac{2 \varepsilon_{n, \mathrm{rms}} u}{k_{0} \beta \gamma}=\frac{q \bar{I}}{8 \pi \varepsilon_{0} m c^{2} \gamma^{3} \beta^{2} \ell f k_{0}^{2}},
$$

which is independent of the input emittance. Substituting the result of Eq. (9) into Eq. (6), the model predicts that, for a given value of $\mu$, the maximum transverse halo amplitude increases with increasing beam current and with decreasing frequency, bunch length, and focusing strength. The emittance-dominated limit corresponds to $u \ll 1$, and we find

$$
a^{2} \cong \frac{\varepsilon_{n, \mathrm{rms}}}{k_{0} \beta \gamma}\left[1+\frac{q \bar{I}}{16 \pi \varepsilon_{0} m c^{2} \gamma^{2} \beta \ell f k_{0} \varepsilon_{n, \mathrm{rms}}}\right] .
$$

In this limit the second term is much less than unity, and the model predicts that the maximum amplitude increases mainly with increasing emittance and with decreasing focusing strength.

\section{SUMMARY}

The particle-core model describes the transverse dynamics of particles in a beam halo. We use the model to represent a beam whose initial density distribution includes particles that populate a finite size Debye tail at the edge of the beam. The particle-core model makes quantitative predictions about the halo that is formed from the resonant interaction between individual particles in the tail and a mismatch-induced core oscillation. We have expressed the model in a dimensionless form and have solved the nonlinear equations of the model by numerical integration. Over a wide range of mismatch parameter values, the results expressed in dimensionless form are remarkably insensitive to the tune depression and to the core-density distribution. The exception to this is the growth rate of the halo which, for large values of $\eta$, depends strongly on the tune depression, increasing with increasing $\eta$ as the beam becomes more emittance dominated, as shown in Fig. 12. The model predicts that the halo will be limited to a maximum amplitude, which depends on the magnitude of the mismatch parameter. The simulation results, using two different space-charge codes and three different initial particle distributions, agree well with the model predictions for the maximum amplitude. We have obtained a scaling formula for the maximum amplitude and have shown that for a given beam current and energy, the maximum transverse amplitude is reduced by stronger focusing, higher frequency, longer bunch length, and smaller input emittance.

For small values of the tune-depression ratio, $\eta<0.3$, stochastic behavior is observed, which begins along the separatrix and grows with decreasing $\eta$. The main effect of the chaos is not so much to increase the maximum halo amplitude, but to reduce the spacing between the core radius and the inner separatrix. Qualitatively, this can be expected to increase the population of the halo.
Now let us briefly review the assumptions and limitations of the model. The particle-core model is a simple approximation for the evolution of particles in an rmsmismatched beam. To use the model, one integrates the equations of motion of test particles moving in the field of an rms-mismatched core; the core itself is modeled by integrating the rms envelope equations. We assume that the form of the core distribution function does not change and that the core emittance does not change. In a real beam other collective modes in addition to the breathing mode, and other resonances of the particle-core system, may also be present. Charge redistribution in the core may rapidly change the core distribution, an effect that is not included in this non-self-consistent treatment of the core. The complexity of the real situation has been described elsewhere in terms of turbulence models of beam halo [26,27]. Nevertheless, the good agreement of our model with the numerical simulations suggests that this added complexity has a minor effect on the maximum resonant amplitude. We believe that the particle-core model includes the main resonant mechanism that explains how particles in the initial tail of a realistic beam distribution can be driven to larger amplitudes to form a halo. We find that the basic structure of the separatrix in the stroboscopic phase-space plots of the model is nearly the same for both a uniform and a Gaussian core, which suggests that the halo dynamics described by the model is a general property of a mismatched beam that is insensitive to the shape of the core distribution and is not an artifact of any particular core distribution. For most realistic beam distributions, an initial tail exists at the edge of the beam that can easily extend beyond the inner separatrix into the resonance region of the stroboscopic phase-space plot; particles that lie within this outer tail will be resonantly driven to larger amplitudes to form the halo. For the special cases of a K-V distribution or an equilibrium distribution in the extreme space-charge limit, the beam has a uniform cross section with no initial tail. In those cases, an additional mechanism is required to explain how the particles cross the inner separatrix, after which, a halo will develop because of the resonance [28]; such an additional mechanism may involve other collective modes. In this paper we have studied transverse dynamics under the assumption that the external focusing force is linear. When we track large amplitude particles beyond the outer separatrix, we see bounded betatronlike motion in a field dominated by the linear focusing field. The longitudinal dynamics for a bunched beam, which is not treated by our model, is being studied using a similar particle-core model based on a spheroidal bunch geometry $[22,28]$. The inclusion of nonlinear rf forces in that model may lead to unbounded motion for some particles.

Once the expected beam mismatch is estimated, the particle-core model may be used to estimate the maximum transverse amplitude of the beam halo and a suitable aperture size can be chosen that will reduce the threat of beam loss from the halo. In determining the expected 
beam mismatch for a high-current linac design, one must account for the fact that in a real linac the beam may be mismatched as a result of a large number of small parameter errors rather than from a single error as is described by the particle-core model. Thus, a realistic determination of the mismatch and the halo amplitude for a given linac design should be made by doing multiparticle simulation studies including random errors based on realistic expectations.

\section{ACKNOWLEDGMENTS}

The authors acknowledge support from the U.S. Department of Energy. They thank Robert Gluckstern, Ingo Hofmann, James O'Connell, and Martin Reiser for helpful discussions.

[1] H. Koziol, Los Alamos MP Division Report, MP-3-75-1, 1975.

[2] D. Kehne, M. Reiser, and H. Rudd, in Proceedings of the IEEE 1991 Particle Accelerator Conference, 1991, edited by L. Lizama and J. Chew (IEEE, New York, 1991), p. 248 .

[3] P.W. Lisowski, in Proceedings of the 1997 Particle Accelerator Conference, Vancouver, British Columbia, Canada (IEEE, Piscataway, NJ, 1998), p. 3780.

[4] T.P. Wangler, K. R. Crandall, R. S. Mills, and M. Reiser, IEEE Trans. Nucl. Sci. 32, 2196 (1985).

[5] J.D. Lawson, The Physics of Charged Particle Beams (Clarendon Press, Oxford, 1988), 2nd ed.

[6] J. Struckmeier and M. Reiser, Part. Accel. 14, 227 (1984).

[7] A. Cucchetti, M. Reiser, and T.P. Wangler, in Proceedings of the IEEE 1991 Particle Accelerator Conference, 1991, edited by L. Lizama and J. Chew (IEEE, New York, 1991), p. 251.

[8] M. Reiser, Proceedings of the IEEE 1991 Particle Accelerator Conference, 1991, edited by L. Lizama and J. Chew (IEEE, New York, 1991), p. 2497.

[9] T.P. Wangler, in Computational Accelerator Physics, edited by Robert Ryne, AIP Conf. Proc. No. 297 (AIP, New York, 1994), p. 9.
[10] O. A. Anderson and L. Soroka, 1987 Particle Accelerator Conference, 1987, edited by E. R. Lindstrom and L.S. Taylor (IEEE, New York, 1987), p. 1043.

[11] J. S. O'Connell, T.P. Wangler, R.S. Mills, and K. R. Crandall, in Proceedings of the 1993 Particle Accelerator Conference, 1993, edited by S.T. Corneliussen (IEEE, New York, 1993), p. 3657.

[12] R. Gluckstern, Phys. Rev. Lett. 73, 1247 (1994).

[13] J. Lagniel, Nucl. Instrum. Methods Phys. Res., Sect. A 345, 46 (1994).

[14] J. Lagniel, Nucl. Instrum. Methods Phys. Res., Sect. A 345, 405 (1994).

[15] T. P. Wangler, Los Alamos Report LA-UR-94-1135, 1994.

[16] R. Jameson, Los Alamos Report LA-UR-93-1209, 1994.

[17] R. Ryne, S. Habib, and T.P. Wangler, AIP Conf. Proc. 346, 383 (1994).

[18] R. L. Gluckstern, W.H. Cheng, and H. Ye, Phys. Rev. Lett. 75, 2835 (1995).

[19] Robert L. Gluckstern, Wen-Hao Cheng, Sergey S. Kurennoy, and Huanchan Ye, Phys. Rev. E 54, 6788 (1996).

[20] T.P. Wangler, R. W. Garnett, E. R. Gray, R. D. Ryne, and T.S. Wang, in Proceedings of XVIII International Linear Accelerator Conference, Geneva, Switzerland, 1996, edited by C. Hill and M. Vretenar (CERN, Geneva, 1996), p. 372.

[21] Robert L. Gluckstern and Sergey S. Kurennoy, in Proceedings of the 1997 Particle Accelerator Conference, Vancouver, British Columbia, Canada (IEEE, Piscataway, NJ, 1998), p. 1950.

[22] J. Barnard and S. Lund, in Proceedings of the 1997 Particle Accelerator Conference, Vancouver, British Columbia, Canada (IEEE, Piscataway, NJ, 1998), p. 1929.

[23] T.P. Wangler, E. R. Gray, S. Nath, and K. R. Crandall, in Proceedings of the 1997 Particle Accelerator Conference, Vancouver, British Columbia, Canada (IEEE, Piscataway, NJ, 1998), p. 915.

[24] H. Okamoto and M. Ikegami, Phys. Rev. E 55, 4694 (1997).

[25] K. R. Crandall (unpublished); a description of SCHEFF may be found in T.P. Wangler, RF Linear Accelerators (John Wiley \& Sons, New York, 1998), pp. 270-272.

[26] O. A. Anderson, Part. Accel. 21, 197 (1987).

[27] C. L. Bohn, Phys. Rev. Lett. 70, 932 (1993).

[28] R. L. Gluckstern, A. V. Fedotov, S. S. Kurrenoy, and R. D. Ryne, Phys. Rev. E 58, 4977 (1998). 